\title{
LEUKOCYTOSIS DURING FIRST FOUR WEEKS OF LIFE IS AN INDEPENDENT RISK FACTOR FOR RETINOPATHY OF PREMATURITY
}

\author{
Pia Lundgren ${ }^{1}$, Susanna Klevebro2,3, Aldina Pivodic ${ }^{4}$, Petter Brodinn,6, Lois E H Smith7, Boubou Hallberg' ${ }^{2,6}$, \\ Ann Hellström ${ }^{1}$ \\ ${ }^{1}$ Section for Ophthalmology, Department of Clinical Neuroscience, Institute of Neuroscience and Physiology, Sahlgrenska Academy, University of \\ Gothenburg, Gothenburg, Sweden \\ 2Department of Clinical Science, Intervention and Technology, Karolinska Institutet, Stockholm, Sweden \\ 'Sachs' Children and Youth Hospital, South General Hospital, Stockholm, Sweden \\ ${ }^{4}$ Statistiska konsultgruppen, Gothenburg, Sweden \\ ${ }^{5}$ Science for Life Laboratory, Department of Women's and Children's Health, Karolinska Institutet, Stockholm, Sweden \\ ${ }^{6}$ Department of Neonatology, Karolinska University Hospital, Stockholm, Sweden \\ ${ }^{7}$ Department of Ophthalmology, Boston Children's Hospital, Harvard Medical School, Boston, MA, USA
}

\begin{abstract}
Aim
This study retrospectively evaluated longitudinal white blood cell (WBC) counts, C-reactive protein (CRP), prenatal and neonatal morbidities as risk factors for severe retinopathy of prematurity (ROP) requiring treatment in a Swedish cohort of very preterm infants.
\end{abstract}

\section{Background}

ROP pathogenesis is multifactorial and ROP development may involve prenatal and neonatal inflammation. A markedly elevated WBC count, leukocytosis, may indicate an inflammatory response.

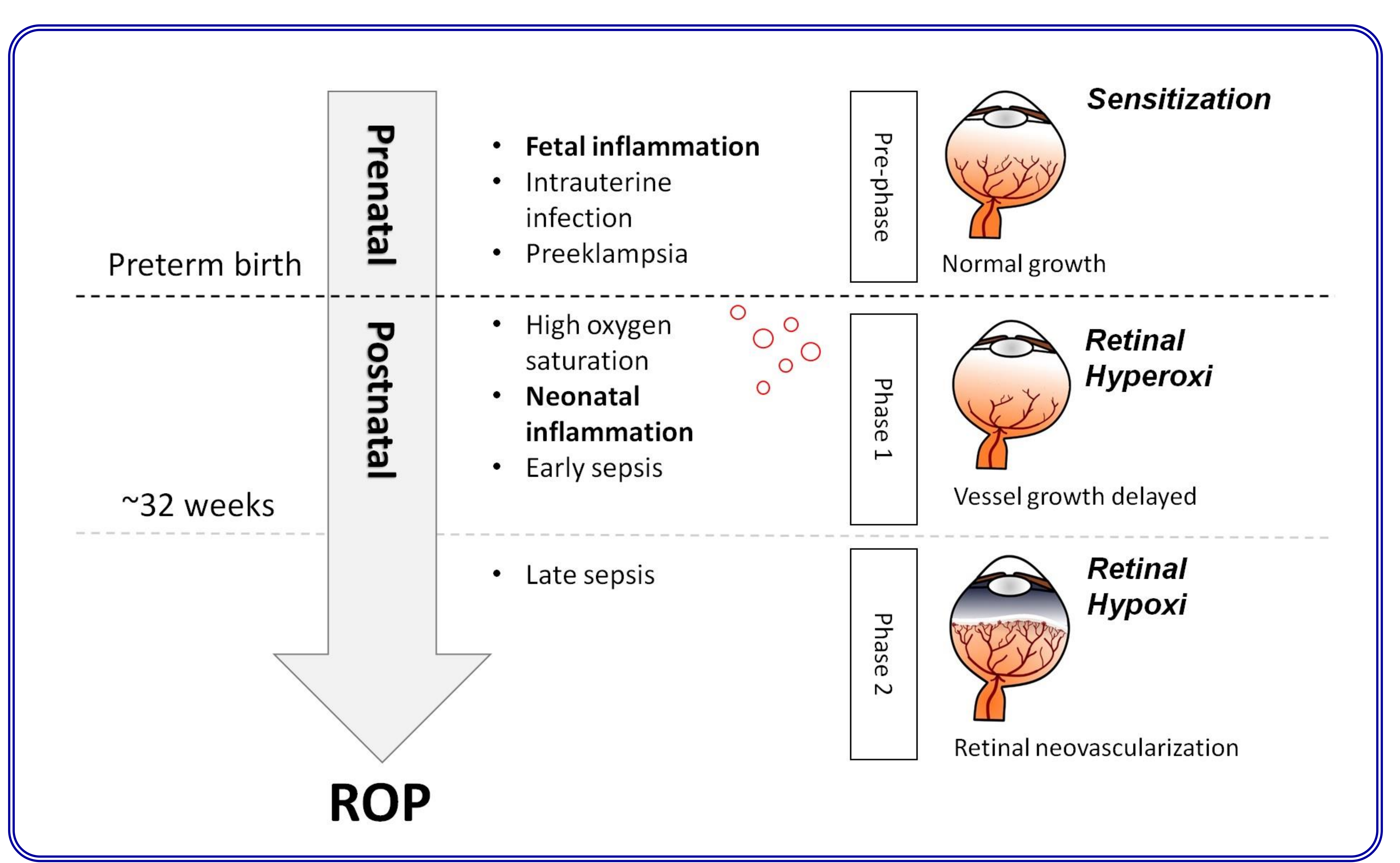

Study subjects and methods

We studied 164 infants born $<27$ weeks gestational age (GA) and screened for ROP in 2008-2011 with a mean (SD) GA at birth of 25.4 (1.0) weeks and mean (SD) birth weight of 799 (160) grams, $18 \%$ (30) were treated for severe ROP.

- Prenatal and neonatal morbidities were recorded.

- Longitudinal WBC and CRP count up to 32 weeks postmenstrual age were reviewed.

- Leukocytosis was defined as $\mathrm{WBC} \geq 30 \times 10^{9} / \mathrm{L}$.

This work was supported by De blindas vänner, Cronquist stiftelse, Carmen and Bertil Regner's Foundation. NIH EY024864, EY017017, BCH IDDRC, 1U54HD090255 P01 HD18655, Lowy Medical Research Institute, European Commission FP7 project 305485 PREVENT-ROP

\section{www.rop.gu.se}

Conclusions

- Leukocytosis during the first four weeks of life was an independent risk factor for severe ROP requiring treatment in extremely preterm infants.

Reducing inflammation in extremely preterm infants might reduce their risk of ROP development.

Results 1: Elevated WBC levels associated with ROP treatment Mean WBC levels was higher in the second week of life $(16.7 \times$ $10^{9} / \mathrm{L}$ vs $24.3 \times 10^{9} / \mathrm{L}$, in infants requiring ROP treatment compared to infants not requiring treatment $(\mathrm{p}=0.00084)$, Figure 1 .

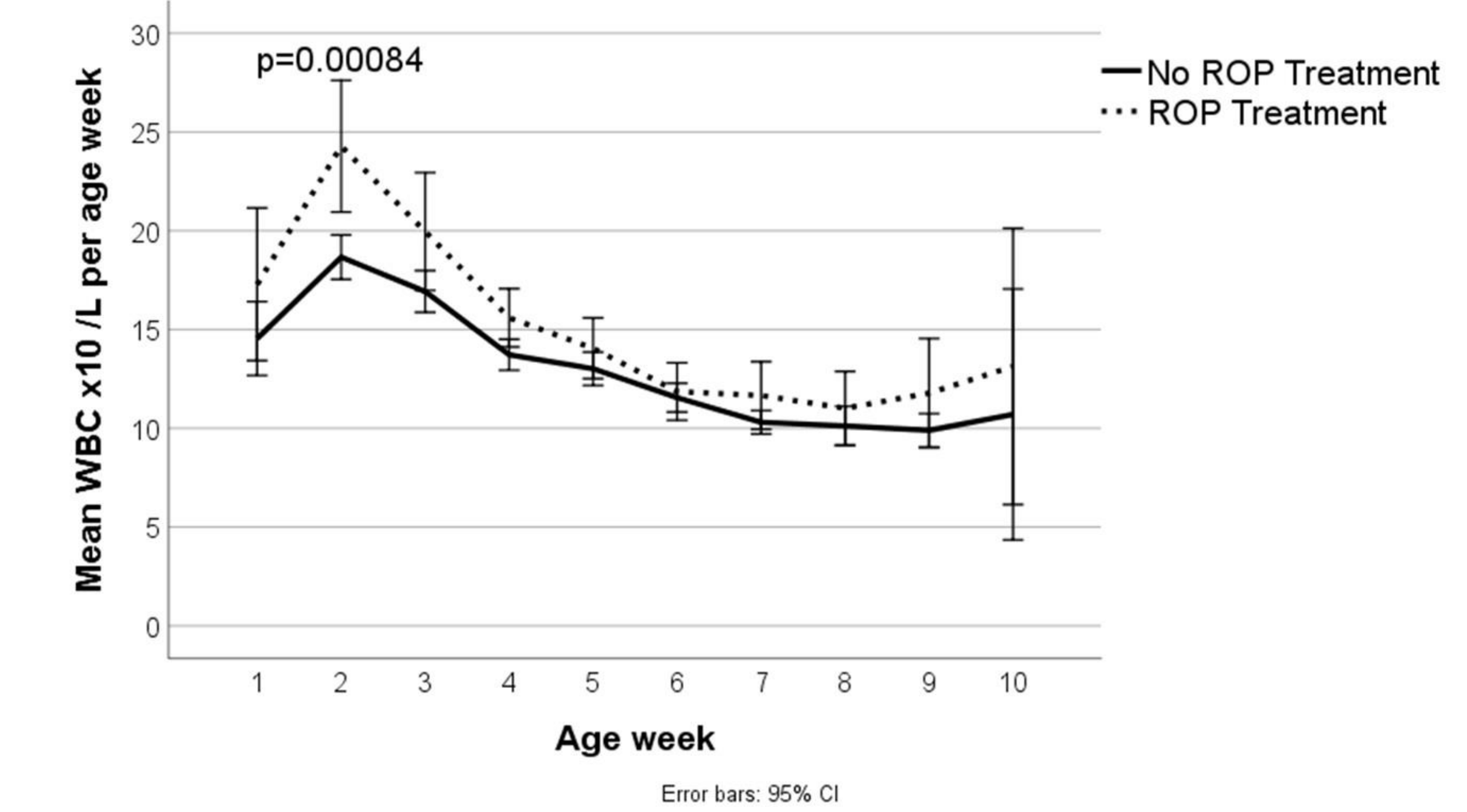

Result 2: Leukocytosis was a risk factor for ROP treatment Leukocytosis (WBC $\geq 30 \times 10^{9} / \mathrm{L}$ ) during first four weeks was more often present in infants requiring ROP treatment (67\% vs $34 \%$, $\mathrm{p}=0.0018$ ).

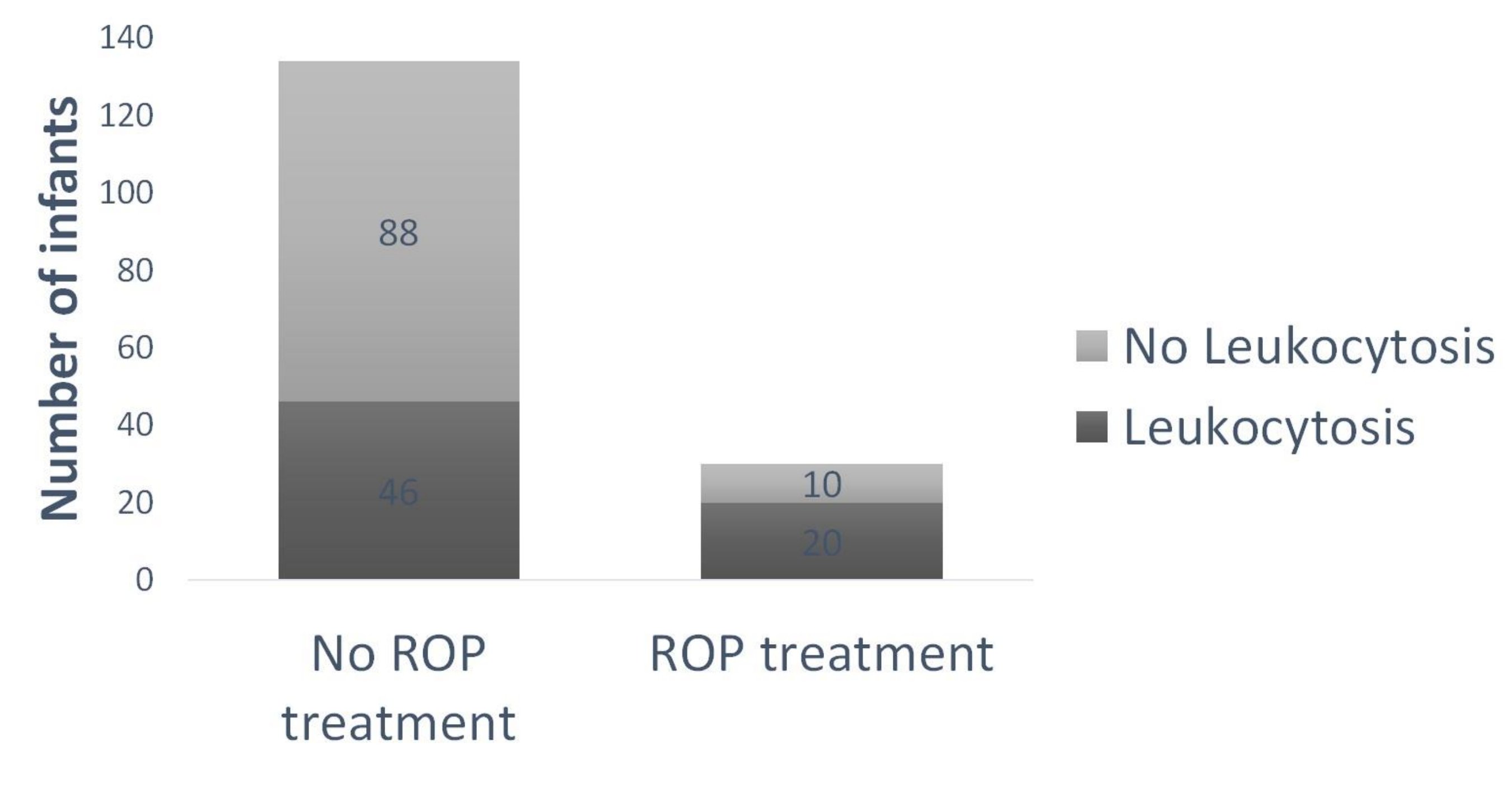

- ROP treatment was positively associated with leukocytosis during the first four weeks of life (OR 3.96, 95\% CI 1.71-9.16, $\mathrm{p}=0.0013$ ) but elevated CRP count were not associated.

- In multivariable logistic regression analyses: leukocytosis (OR $2.83,95 \%$ CI 1.16-6.92, p =0.022) and days of mechanical ventilation during first four weeks of life (OR 1.06, 95\% CI 1.00$1.12, \mathrm{p}=0.034)$, were found to be independent risk factors for ROP treatment, adjusted for GA week. 